\title{
Structurally and morphologically engineered chalcogenide materials for optical and photonic devices
}

\author{
Casey M. Schwarz, ${ }^{\text {a,* }}$ Stephen M. Kuebler $\odot,{ }^{\text {b,c }}$ Clara Rivero-Baleine, ${ }^{d}$ \\ Brandon Triplett, ${ }^{d}$ Myungkoo Kang, ${ }^{c}$ Quentin Altemose, ${ }^{a}$ Cesar Blanco, \\ Kathleen A. Richardson, ${ }^{\mathrm{c}}$ Qingyang Du $\odot,{ }^{\mathrm{e}}$ Skylar Deckoff-Jones, \\ Juejun Hu, ${ }^{\mathrm{e}}$ Yifei Zhang, ${ }^{\mathrm{e}}$ Ying Pan ${ }^{\circ},{ }^{\mathrm{e}}$ and Carlos Ríos $\odot^{\mathrm{e}}$ \\ ${ }^{a}$ Ursinus College, Physics and Astronomy, Collegeville, Pennsylvania, United States \\ ${ }^{b}$ University of Central Florida, Chemistry Department, Orlando, Florida, United States \\ ${ }^{c}$ University of Central Florida, The College of Optics and Photonics, Orlando, Florida, \\ United States \\ ${ }^{\mathrm{d}}$ Lockheed Martin Corporation, Orlando, Florida, United States \\ ${ }^{\mathrm{e}}$ Massachusetts Institute of Technology, Department of Materials Science and Engineering, \\ Cambridge, Massachusetts, United States
}

\begin{abstract}
We discuss several recent advances in the development of methodologies and techniques used to structurally and morphologically engineer chalcogenide $(\mathrm{ChG})$ materials. We introduce two ChG patterning techniques both offering spatial resolution beyond the classical single-photon diffraction limit: multiphoton lithography and thermal scanning probe lithography (TSPL). The former was applied to produce nanoscale modifications in thermally deposited $\mathrm{As}_{2} \mathrm{~S}_{3}$, and we realized gradient refractive index (GRIN) effective medium lens fabrication in multilayer $\mathrm{As}_{2} \mathrm{~S}_{3}-\mathrm{As}_{2} \mathrm{Se}_{3}$ films with features as small as $120 \mathrm{~nm}$ using this approach. The GRIN lens was shown to be optically functional. ChG Ge-Sb-Se-Te (GSST) material was also explored for its potential as a phase-change material (PCM). We demonstrated nanoscale feature patterning using TSPL in PCMs with critical dimensions below $100 \mathrm{~nm}$. In addition, new patterning methods, we also report solution processing of GSST PCMs as an alternative route for ChG film deposition. These new material processing and structuring techniques will offer new pathways for creating functional planar optical and photonic devices. (C) The Authors. Published by SPIE under a Creative Commons Attribution 4.0 Unported License. Distribution or reproduction of this work in whole or in part requires full attribution of the original publication, including its DOI. [DOI: 10 .1117/1.JOM.1.1.013502]
\end{abstract}

Keywords: chalcogenide glass; multiphoton lithography; thermal scanning probe lithography; infrared gradient refractive index.

Paper 20009 received Sep. 10, 2020; accepted for publication Dec. 15, 2020; published online Jan. 26, 2021.

\section{Introduction}

Chalcogenide glasses (ChGs) are formed when one or more chalcogens ( $\mathrm{S}, \mathrm{Se}$, or $\mathrm{Te}$ ) are meltprocessed with one or more elements from groups XIII to XV. ${ }^{1}$ They are well known for their useful optical properties, which has led to many infrared (IR) applications in the fields of medicine, photonics, environmental sensing, and security. ${ }^{2}$ ChGs can be compositionally tuned and processed to have useful properties such as a tailorable coefficient of thermal expansion, a suitably large refraction index or dispersion modification, combined with the ability to maintain a low thermo-optical coefficient $(d n / d T)$, increased mechanical strength, and improved radiation hardness. ${ }^{3}$ Compositions can be crystalline, as in the mineral orpiment arsenic trisulfide $\left(\mathrm{As}_{2} \mathrm{~S}_{3}\right)$, but many form IR-transparent, homogeneous optical-quality glasses (in select cases transformable to optical glass ceramics), that can be engineered to be thermodynamically and/or kinetically stable over a wide range of compositional variations. ${ }^{4,5} \mathrm{ChGs}$ can be used as bulk, fiber, or as thin films via thermal deposition or solution processing. ${ }^{4}$

*Address all correspondence to Casey M. Schwarz, cschwarz@ursinus.edu 
This paper discusses recent advances in the development of methodologies and techniques used to structurally and morphologically engineer $\mathrm{ChG}$ thin films for optical and photonic device applications. Multiphoton lithography (MPL) is used for nanoscale modifications in thermally deposited ChG multicomponent compositions such as $\mathrm{As}_{2} \mathrm{~S}_{3}$. Gradient refractive index (GRIN) effective medium lenses were created in $\mathrm{As}_{2} \mathrm{~S}_{3}$ and characterized for optical function. Laser writing in Ge-Sb-Se-Te (GSST) phase-change materials (PCMs) allows for simple area-selective phase transitions; however, its resolution is bound by the optical diffraction limit. ${ }^{6,7}$ An alternative atomic force microscopy (AFM)-related technique, thermal scanning probe lithography (TSPL) is introduced for high-resolution lithography. TSPL and large area thermal conversion are used for large and nanoscale modifications in GSST PCMs. Processing and characterization of high-performance solutions derived GSST PCMs are demonstrated. Switching performance and prospects of this system as a functional metalens are explored.

\section{Multiphoton Lithography in Arsenic Sulfide $\mathrm{As}_{2} \mathrm{~S}_{3}$ Films}

$\mathrm{As}_{2} \mathrm{~S}_{3}$ is a ChG material with excellent IR transparency $(620 \mathrm{~nm}$ to $11 \mu \mathrm{m})$, an optical band gap at $2.35 \mathrm{eV}(\lambda \sim 517 \mathrm{~nm})$, and large nonlinear refractive indices. ${ }^{8,9}$ Thermal deposition of bulk $\mathrm{As}_{2} \mathrm{~S}_{3}$ fragments the network solid, creating molecular clusters that can be deposited as photosensitive thin films. ${ }^{8}$ Photoexcitation through processes such as the MPL (laser writing) crosslinks the homopolar bonds in the film back to the glassy network solid. ${ }^{8-11}$ Laser written areas remain intact after being submersed in a polar solvent [typically a mixture of diisopropanolamine (DIPA) and dimethyl sulfoxide (DMSO)] while the rest of the unexposed $\mathrm{As}_{2} \mathrm{~S}_{3}$ film is etched away, resulting in a remaining structure that has the shape of the laser written pattern. ${ }^{8-11}$ Although other lithographic techniques for patterning $\mathrm{As}_{2} \mathrm{~S}_{3}$ are available, MPL is the standout method for achieving highly controllable $\mathrm{As}_{2} \mathrm{~S}_{3}$ material processing at the microscale without the use of clean rooms and masks. ${ }^{12}$ Due to its nonlinear optical nature in the laser-matter interaction when ultra-short laser pulses are used, MPL can achieve material modifications that are confined to the focal volume and can reach the micro-and nanoscales without adverse thermal effects. ${ }^{12,13}$ MPL in thermally deposited $\mathrm{As}_{2} \mathrm{~S}_{3}$ films has been shown to produce high-precision three-dimensional (3D) micro- and nanostructures, such as waveguides, woodpile photonic crystals, and nanowires. ${ }^{8,11,14}$ In Sec. 2.1, we show how the optimization of processing conditions is used to photopattern $3 \mathrm{D}$ microstructures in 4- $\mu$ m-thick $\mathrm{As}_{2} \mathrm{~S}_{3}$ films thermally deposited on a ZnSe substrate. ${ }^{10}$ In Sec. 2.2, we use MPL to fabricate $\mathrm{As}_{2} \mathrm{~S}_{3}$ microstructure-based metalenses that offer reduced size, weight, and cost as compared to traditional bulk IR counterparts. ${ }^{8}$

\subsection{Optimization of MPL and Chemical Etch Processing Conditions for the Creation of Microstructures in $\mathrm{As}_{2} \mathrm{~S}_{3}$ Films}

A femtosecond Mai Tai Ti:sapphire laser centered at $800 \mathrm{~nm}$ with a repetition frequency of $80 \mathrm{MHz}$ is used for MPL in 4- $\mu \mathrm{m}$-thick $\mathrm{As}_{2} \mathrm{~S}_{3}$ films thermally deposited on a ZnSe substrate. The beam is passed through a variable attenuator to control the average power and a shutter, both of which are controlled externally though a computer. The beam is focused by either a $50 \times / 0.55$ NA or $100 \times / 0.75$ NA objective onto the $\mathrm{As}_{2} \mathrm{~S}_{3}$ film. The substrate is secured onto a Physik Instrumente H-840 6-Axis Hexapod (programmable stage), and a camera is used to image the sample plane during irradiation. The entire system is controlled by a LabVIEW program that automates the laser writing of predetermined patterns.

A series of experiments were initially conducted in order to dial in on the optimal power, write velocity, and focus depth of laser into films. Dose arrays of raster scans, grids, and concentric circles are laser written into the film at varying average powers, write velocities, and focus depths of laser into film to test for the best etch selectivity as a function of these parameters. Examples of these laser written patterns are shown in Fig. 1. A range of powers is defined as a percentage of maximum possible average power at the sample plane from the variable attenuator. For the $50 \times / 0.55$ NA objective, $100 \%$ power corresponds to $40 \mathrm{~mW}$ and for the $100 \times / 0.75$ NA objective, $100 \%$ power corresponds to $35 \mathrm{~mW}$. The etch results from these experiments indicate that a write velocity of $0.25 \mathrm{~mm} / \mathrm{s}$ at an average power in the $40 \%$ to $60 \%$ range induces the desired 
(a)

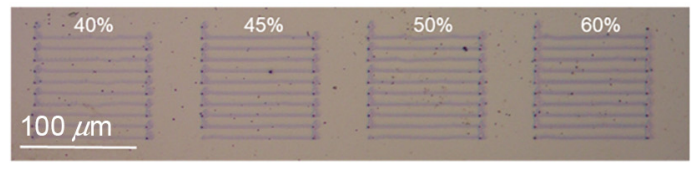

(b)

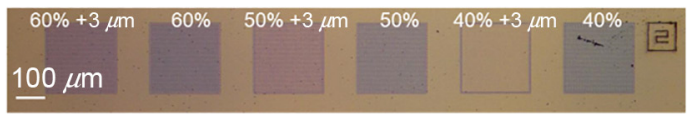

(c)

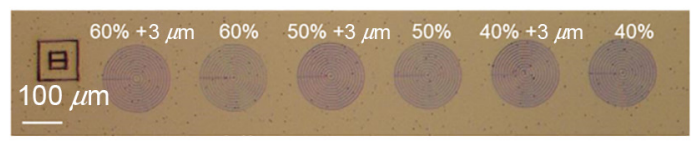

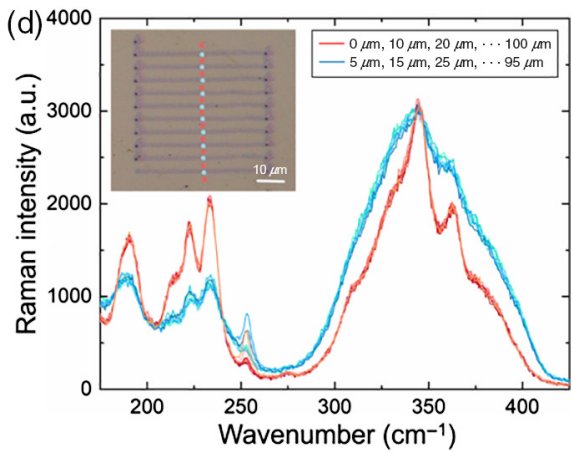

Fig. 1 (a) Raster scan dose array with varying power, indicated by the percentages. 10- $\mu \mathrm{m}$ spacing, $0.25 \mathrm{~mm} / \mathrm{s}$ write velocity, and 50x objective. (b) Grid dose array with varying power and depth of focus. " $+3 \mu \mathrm{m}$ " indicates the laser was focused $3 \mu \mathrm{m}$ into the film. $5-\mu \mathrm{m}$ spacing, $0.25 \mathrm{~mm} / \mathrm{s}$ write velocity, and $100 \times$ objective. (c) Same as (b) except the pattern is now a group of concentric circles. (d) Raman spectra graph for the region shown in the embedded image. The microscope image (20x magnification) is of a $10-\mu \mathrm{m}$ spaced raster scan irradiated at $60 \%$ power and $0.25 \mathrm{~mm} / \mathrm{s}$ write velocity with a $50 \times$ objective. Each red spectrum is measured from the asdeposited film, whereas each blue spectrum is measured from a laser exposed region.

molecular change to the film without ablation. Higher powers and lower write velocities were usually found to ablate the film, whereas lower powers and higher write velocities did not result in good contrast between exposed and unexposed regions after etching. Further experimentation is in progress to determine the depth of focus into film for optimal etch selectivity, as indicated by the $+3-\mu \mathrm{m}$ legend in Fig. 1. In this scenario, the laser is focused 3- $\mu \mathrm{m}$ deep into the film.

The postirradiation molecular structure of the film was investigated using micro-Raman spectroscopy. Figure 1(d) shows the Raman spectra collected at 5- $\mu$ m intervals over a raster scan ( $60 \%$ power, $0.25 \mathrm{~mm} / \mathrm{s}$ write velocity). A Horiba Jobin Yvon confocal micro-Raman system with an excitation wavelength of $785 \mathrm{~nm}$ was used. The beam was focused by a $100 \times / 0.75 \mathrm{NA}$ objective and the Raman signal was collected for $60 \mathrm{~s}$ at a low optical power (reduced by optical density filter), to prevent any undesired photoinduced change to the film. The laser-induced film cross-linking causes the exposed regions of the film to exhibit a different Raman signature from the surrounding pristine film. ${ }^{11,13}$ Most notably, the $\mathrm{As}_{4} \mathrm{~S}_{4}$ molecular clusters that are present in the as-deposited/unirradiated film, characterized by the Raman peaks at 222 and $363 \mathrm{~cm}^{-1} 11,13$ [as illustrated by the red spectra of Fig. 1(d)] emerge during the laser exposure process (blue spectra), leading to good etch selectivity between these two regions.

After the patterns are written using the laser, the sample is submerged into a solvent mixture of DIPA and DMSO for the etching process. After the sample is initially immersed for an hour, the resulting structure is analyzed using a ZYGO 6300 white-light interferometer (Fig. 2). Subsequent etching is done in 30-min increments until the profile of the structure is fully developed. Etch times vary depending on laser power and target resolution of laser-written structures, but it typically takes between 1 to $4 \mathrm{~h}$ of immersion. Good etch selectivity has been shown repeatedly down to 7.5- $\mu \mathrm{m}$ feature spacing, as shown in Fig. 2(a). The pictured 7.5- $\mu \mathrm{m}$ grid structure in Fig. 2(a) (irradiated at $60 \%$ power or $21 \mathrm{~mW}, 0.25 \mathrm{~mm} / \mathrm{s}$, and $100 \times$ objective) was achieved with $1 \mathrm{~h}$ of etching. At $5-\mu \mathrm{m}$ feature spacing, etch selectivity takes longer to develop. This can be attributed to undesired partial exposure of the film in the regions between the laser write paths.

The height map shown in Fig. 2(b) indicates that the structure did not fully resolve after $1 \mathrm{~h}$ of etching. Subsequent etching on this sample resulted in the desired structure, as evident by the height map in Fig. 2(c). This 5- $\mu \mathrm{m}$-spaced structure (irradiated at $60 \%$ power or $21 \mathrm{~mW}$, $0.25 \mathrm{~mm} / \mathrm{s}$, and $100 \times$ objective) took $240 \mathrm{~min}$ of etching to fully resolve.

In summary, MPL is shown to be effective technique for photopatterning 4- $\mu \mathrm{m}$-thick thermally deposited $\mathrm{As}_{2} \mathrm{~S}_{3}$ films. Experimentation through repeated dose arrays indicate that an average irradiation power between $40 \%$ and $60 \%$ at a write speed of $0.25 \mathrm{~mm} / \mathrm{s}$ induces the desired molecule change in the films. Good etch selectivity for these conditions were shown repeatedly for $7.5-\mu \mathrm{m}$ feature spacing after $1 \mathrm{~h}$ of etching and for $5-\mu \mathrm{m}$ feature spacing after $240 \mathrm{~min}$ of etching. 
(a)

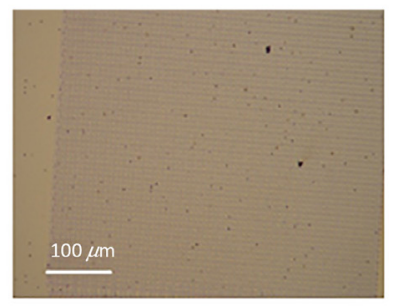

(b)

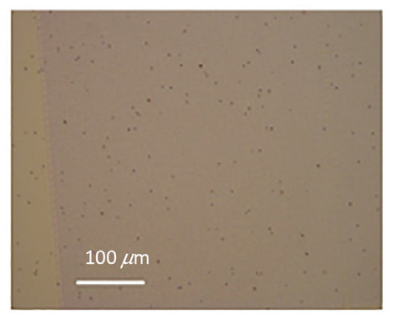

(c)

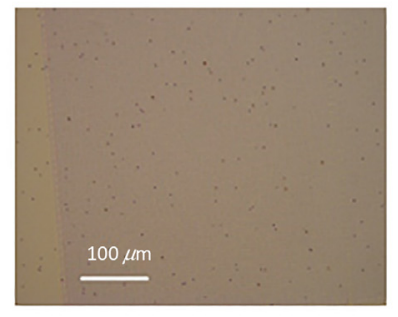

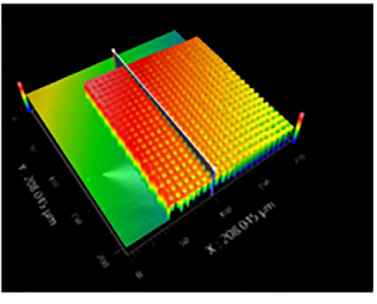
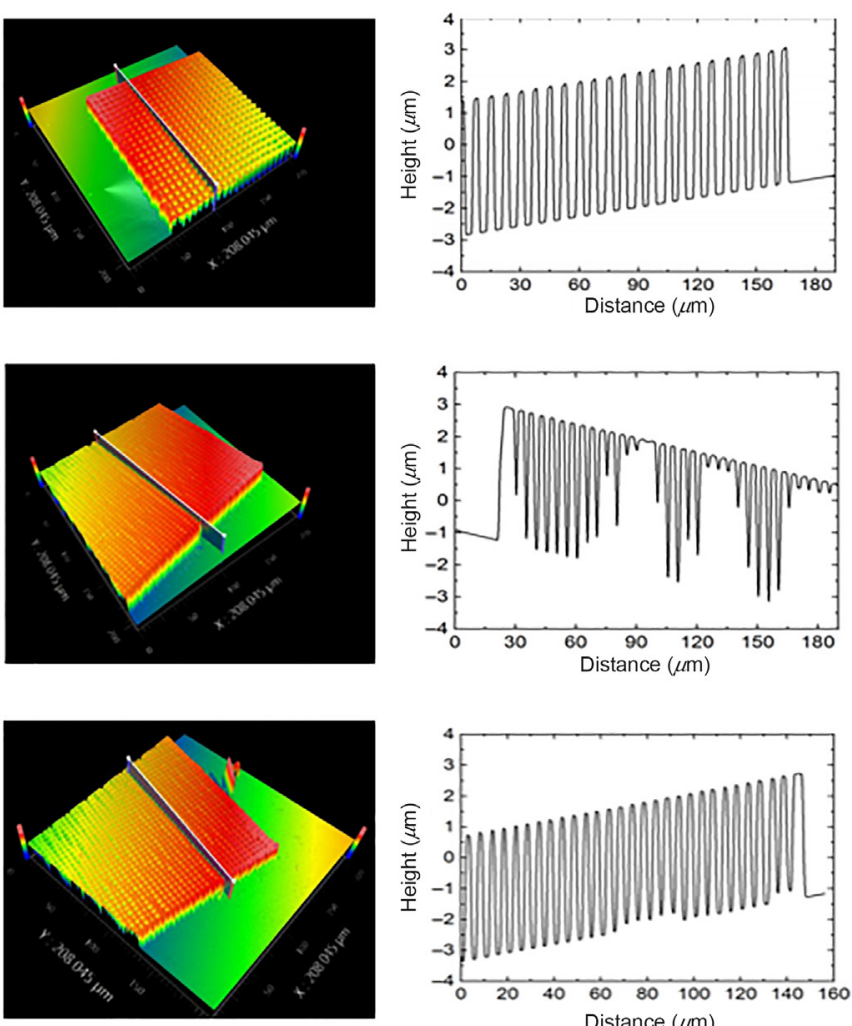

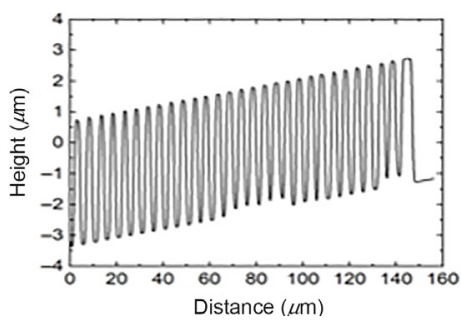

Fig. 2 (a) $7.5-\mu \mathrm{m}$ spacing grid after $1 \mathrm{~h}$ of etching with its respective height map. (b) $5-\mu \mathrm{m}$ spacing grid after $1 \mathrm{~h}$ of etching, with its height map that indicates it is not fully resolved. (c) Same grid in (b) after 240 min of etching, showing that is has become fully resolved.

\subsection{Multiphoton Lithography of Functional GRIN Lenses}

Single-layer $\mathrm{As}_{2} \mathrm{~S}_{3}$ films are inadequate for nanostructure MPL, where the incident wavelength is on the order of, or smaller than, the thickness of the film, because of the standing wave caused by reflection at the substrate interface. ${ }^{15}$ This effect leads to the production of unintended nanobead structures with large sizes and poor substrate adhesion. In this section, we investigated the properties and processing of MPL in single- and multilayered films of $\mathrm{As}_{2} \mathrm{~S}_{3}$ thermally deposited onto an underlying thin film of arsenic selenide $\left(\mathrm{As}_{2} \mathrm{Se}_{3}\right)$ and the creation and optical characterization of a GRIN effective medium lens.

Thermal deposition was used to create a multilayered film of $\mathrm{As}_{2} \mathrm{~S}_{3}$ on an anti-reflective (AR) layer of $\mathrm{As}_{2} \mathrm{Se}_{3}$. A layer of $\mathrm{As}_{2} \mathrm{Se}_{3}$ was deposited first on polished silicon wafers followed by thermal evaporation of a layer of $\mathrm{As}_{2} \mathrm{~S}_{3}$. The thermal deposition process has been described previously. ${ }^{8,9,15,16} \mathrm{The}_{\mathrm{As}} \mathrm{S}_{3}$ layer was deposited at a rate of $30 \AA \mathrm{As}^{-1}$, and the thickness of the resulting film was $896 \pm 13 \mathrm{~nm}$. The $\mathrm{As}_{2} \mathrm{Se}_{3}$ layer was deposited at a rate of $4 \AA \mathrm{s}^{-1}$ and the thickness of the resulting film was $203 \mathrm{~nm} \pm 3 \mathrm{~nm}$. The $\mathrm{As}_{2} \mathrm{Se}_{3}$ serves as an AR layer, which reduces reflection of the incident laser beam back into the focal volume during laser patterning.

MPL was performed using a system and process described previously. ${ }^{17}$ MPL was conducted using the output from a mode-locked Ti:sapphire laser $(\lambda=800 \mathrm{~nm}, 76 \mathrm{MHz}$ repetition rate, and $120 \mathrm{fs}$ pulse duration). An acousto-optic modulator was used for the electronic control of the intensity of the laser beam. The laser pulses were focused into the film with a $100 \times / 1.4$ NA oilimmersion objective (Nikon). GRIN lenses were photopatterned by translating the film along programmed paths in the $x, y$, and $z$ directions at a speed of $50 \mu \mathrm{m} \mathrm{s}^{-1}$ while keeping the laser beam fixed, and then opening and closing a shutter in synchrony with the movements to control local exposure. The average focused laser power was varied from 0.05 to $0.50 \mathrm{~mW}$. The detailed chemical etch processing has been described elsewhere. ${ }^{8,16}$

Incorporating the etch-resistant $\mathrm{As}_{2} \mathrm{Se}_{3}$ AR-layer between the $\mathrm{ChG}$ fabrication layer and the substrate reduces the amplitude of a standing wave caused by reflection at the substrate 
interface $^{15,18}$ This reduction resulted in the generation of pillars with uniformly cylindrical shapes with increased material-substrate adhesion that were more likely to obtain a smaller size before delamination than the bead-shaped features created in the single layered film [Fig. 3(a)]. The uniform shapes also allowed for more predictable fill fractions when compared to stacked bead shaped nanostructures. ${ }^{8,15}$ Predictable fill fractions are important for GRIN lens design when determining effective index and optical function. Large pillar arrays of uniformly cylindrical shapes were demonstrated in the previous works. ${ }^{8,15}$

GRIN effective-medium lenses with diameters of 20 and $100 \mu \mathrm{m}$ were successfully fabricated in the multilayered film. Figure 2(c) shows scanning electron microscope images of the top-down view of the GRIN lens having a diameter of $20 \mu \mathrm{m}$. The inset to Fig. 2(c) shows the top-down view of the GRIN lens having a diameter of $100 \mu \mathrm{m}$.

The GRIN lenses consisted of cylindrical pillar features separated by a pitch of $500 \mathrm{~nm}$. The lenses were designed to have a spherical refractive index profile that ranged from $n=1.06$ to 2.45, the latter representing a region completely filled in with $\mathrm{As}_{2} \mathrm{~S}_{3}$. The corresponding total optical phase delay from center to edge of the lens would be $1.26 \pi$. The index profile was created by fabricating pillars having diameters that decreased with radial distance from the center of the lens, so that the corresponding decrease in local fill-fraction of the high-index $\mathrm{ChG}$ creates a local refractive index that follows that targeted spherical refractive index profile. Across the lens, the pillars had consistent height, and within a small designed region, the diameters of the pillars

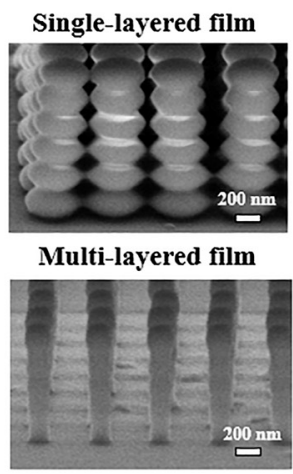

(a)

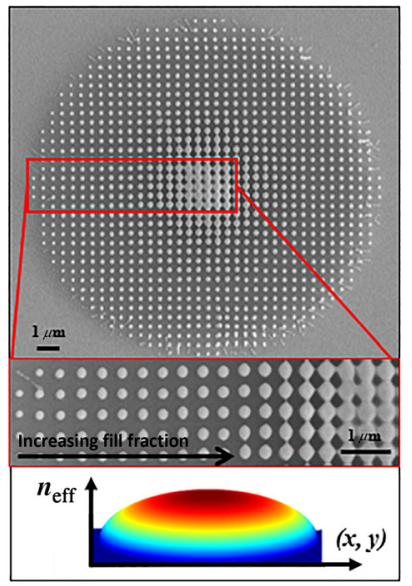

(c)
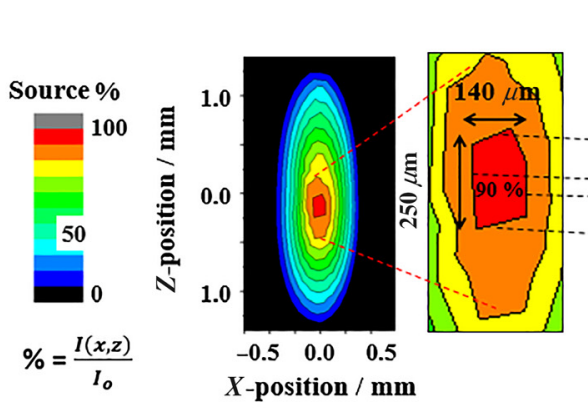

Si substrate

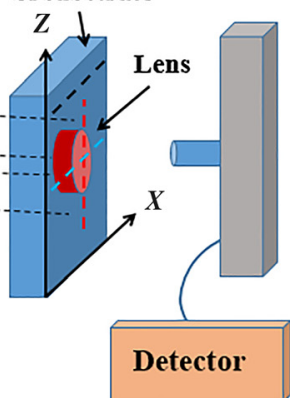

(b)

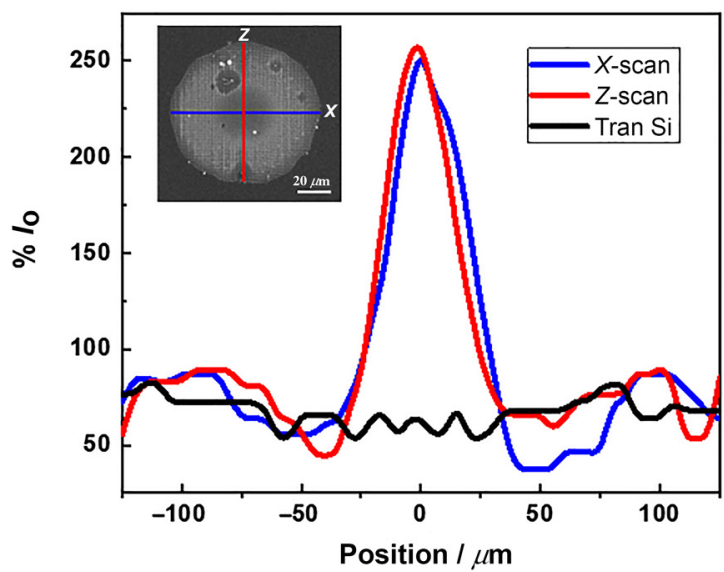

(d)

Fig. 3 (a) Comparison of pillars fabricated in single-layered film (top) and multi-layered film with an AR coating (bottom). (b) Diagram of the source beam interaction with sample. (c) GRIN effectivemedium lens (diameter $=20 \mu \mathrm{m}$ ) designed to have a spherical refractive index profile and fabricated by MPL in thermally deposited $\mathrm{As}_{2} \mathrm{~S}_{3}$. This device consists of cylindrical pillars, which increased in fill fraction (effective refractive index) from the outside diameter to the inner. (d) Transverse intensity scans within focal plane of the GRIN effective medium lens (diameter = $100 \mu \mathrm{m})$ designed to have a spherical refractive index profile. 
were similar. Widths of the pillars ranged from $120 \mathrm{~nm} \pm 14 \mathrm{~nm}$ fabricated at a power of $0.16 \mathrm{~mW}$ to pillars large enough to overfill the entire area. The pillar heights were measured to be a $907 \mathrm{~nm} \pm 10 \mathrm{~nm}$ across the structure and were fixed based by the original film height.

The GRIN lenses were characterized using the output from an Er:YAG laser $(\lambda=2.94 \mu \mathrm{m}$, $170-\mu$ s pulse duration) with the system shown schematically in Fig. 3(b). The laser beam overfilled the sample area so that the intensity distribution across the microlens was uniform. An optical fiber coupled to an IR photodetector (ThorLabs PDA 20H) was mounted on a three-axis translation stage and positioned behind the sample. Transmitted light collected by the fiber provided a relative measure of the local intensity as a function of position behind the lens. Measurements of transmitted light far from the lens provided a baseline measurement that can be used to correct for loss due to reflection from the supporting silicon substrate.

Figure 3(c) shows data from the optical characterization of the $100-\mu \mathrm{m}$ diameter GRIN lens shown in the inset. The black trace is a line scan of light intensity transmitted through the silicon substrate far from the lens. The transmission is roughly $50 \%$, as expected due to reflection losses at both the optical interfaces of the front- and backsides of the silicon substrate. The transmission versus position is uniform within the shot-to-shot variation of the pulsed laser, which confirms that the lens will be uniformly illuminated when positioned in the probe beam. Figure 3(d) shows the transverse intensity scans within the focal plane of the GRIN lens. Irradiance profiles generated by scanning in the $x$ or $z$ direction of the lens resulted in peaks of $\sim 250 \%$ of $I_{0}$.

A homogenous phase shifting aperture "block" with a $100-\mu \mathrm{m}$ diameter was created and characterized in the same manner as the lens. This was done in order to benchmark the transmission of the lens and account for increased throughput on the front-interface of the substrate that may result from refractive-index matching caused by the nanostructures. At the focal point of the lens, the intensity was found to be two times more intense than that transmitted through the uniform block, and four times as intense as light transmitted through the unpatterned substrate away from the lens. The transmitted light behind the lens had a clear focal structure with an FWHM of $32.1 \mu \mathrm{m} \pm 1.9 \mu \mathrm{m}$ for the $x$ scan and $28.6 \mu \mathrm{m} \pm 1.5 \mu \mathrm{m}$ for the $z$ scan. The effective width of the incident beam may be taken to be equivalent to the effective aperture set by the width of the GRIN lens, which was $\sim 140 \times 250 \mu \mathrm{m}^{2}$ at $90 \%$ intensity.

The addition of an AR layer enabled the fabrication of robust reproducible pillars with better material substrate adhesion and smaller dimensions via MPL in $\mathrm{As}_{2} \mathrm{~S}_{3}$ film. GRIN lenses with spherical index profiles were created and IR characterization indicated optical functionality. This work opens new routes to ChG-based electronics and optical devices such as detectors, sensors, photonics waveguides, and acousto-optics.

\section{Processing and Nanopatterning of Chalcogenide Phase Change Materials}

We have shown in preceding sections that MPL is a powerful technique to generate large-area and high-aspect-ratio patterns in $\mathrm{ChG}$ films via modulating the glass local network structures and hence their chemical solubility. Here, we turn our attention to another class of $\mathrm{ChG}$ compounds known as phase change materials (PCMs). PCMs possess an ability to reversibly transform between the amorphous and crystalline states via electrical, ${ }^{19-22}$ optical, ${ }^{23-26}$ thermal, ${ }^{27-30}$ or a combination of these stimuli. ${ }^{31,32}$ The switching property of PCMs has enabled optical storage media devices including rewritable DVDs and Blu-ray discs while also opening the way toward next generation nonvolatile memory applications such as phase-change random access memory. Recently, PCMs are entering the arena of integrated photonic applications as platforms to arbitrarily tailor light-matter interactions.

A ChG alloy of $\mathrm{Ge}_{2} \mathrm{Sb}_{2} \mathrm{Te}_{5}$ is notably the most industrially adopted PCM due to its rapidly transformed response, exhibiting a large contrast in optical and electrical properties upon phase transitions as well as thermal stability over a wide range of temperatures. ${ }^{10-12}$ This unique attribute qualifies PCMs as promising material candidates for innovative reconfigurable optoelectronic devices. ${ }^{28,33-41}$ Therefore, different from $\mathrm{As}_{2} \mathrm{~S}_{3}$ and $\mathrm{As}_{2} \mathrm{Se}_{3}$ whose photosensitive structural changes are more subtle, photonic structures can be directly written into PCM films by inducing crystallization in a spatial-selective manner without resorting to subsequent chemical etching. 
In the following studies, we focus on $\mathrm{Ge}_{2} \mathrm{Sb}_{2} \mathrm{Se}_{5-x} \mathrm{Te}_{x}$ ( $x=0.5$ to 1.0) (GSST), an emerging composition exhibiting exceptional bistate broadband transparency ${ }^{19-22}$ The addition of Se leads to a marked decrease in absorption-induced optical loss while the remaining Te allows the PCM to retain a large contrast in refractive index upon phase transitions. The optical constants of GSST are shown in Fig. 5(a). The ability to produce subwavelength features in GSST is, therefore, of significant interest for applications such as photonic switching and active metasurfaces. ${ }^{42,43}$ Although laser patterning provides a facile route for triggering area-selective phase transition in PCMs, its resolution is ultimately bound by the optical diffraction limit. ${ }^{6,7}$ In Sec. 3.1, we introduce a stylus-based writing technique based on AFM enabling nanoscale pattering in thermally deposited GSST well below the wavelength. In Sec. 3.2, we explore solution processing of GSST materials as an alternative to thermal deposition and as a route to films that can be deposited and patterned on large-area nonplanar platforms.

\subsection{Nanopatterning of Chalcogenide Phase Change Material via Thermal Scanning Probe Lithography}

Stylus-based writing based on AFM techniques offers significantly improved resolution not constrained by optical diffraction. So far, AFM-based patterning of PCMs has been limited to external laser-assisted heating or using conductive atomic force microscopy (c-AFM). ${ }^{44,45}$ However, the c-AFM method is limited to substrates that can form a conductive loop with the AFM tip to

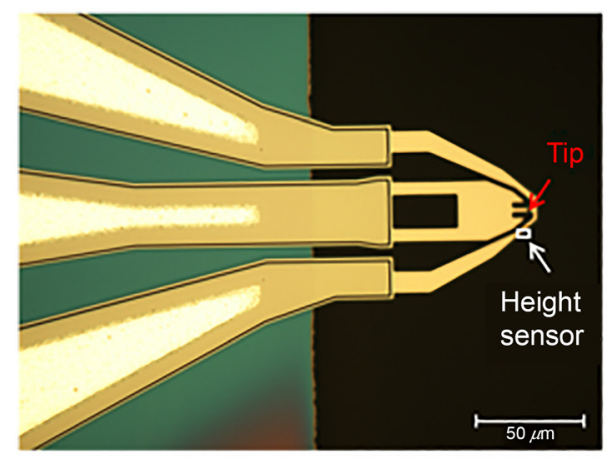

(a)

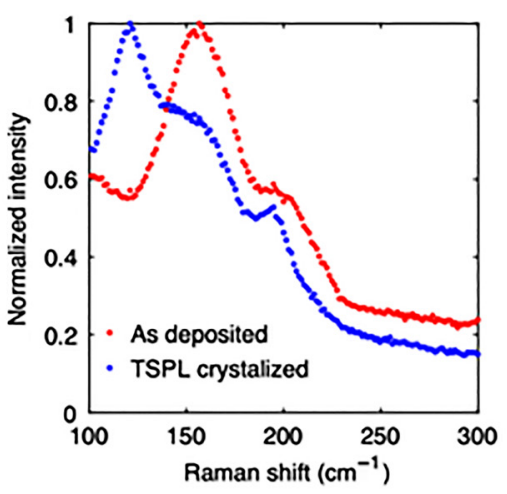

(b)

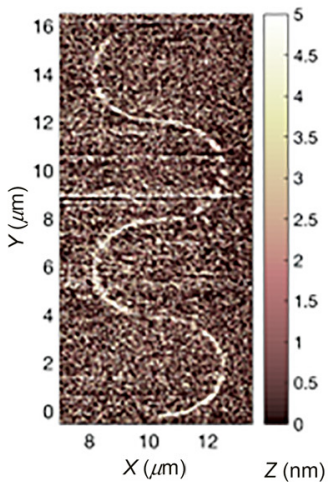

(c)

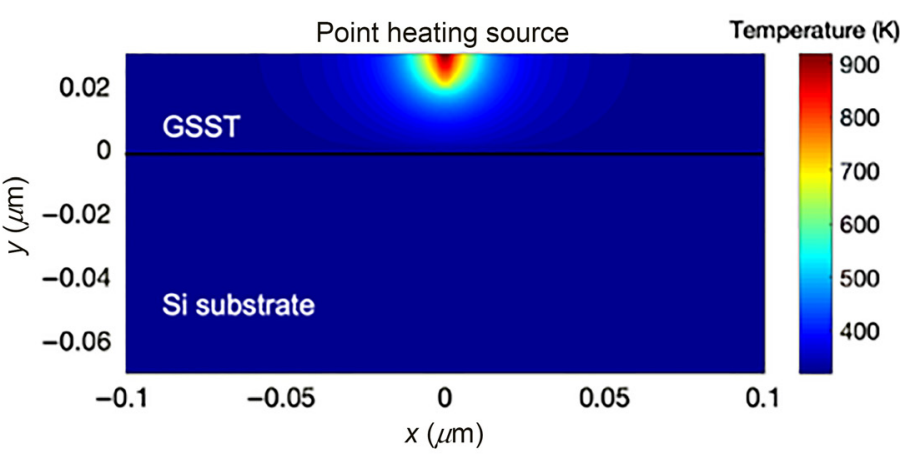

(d)

Fig. 4 Thermal scanning probe patterning of the phase-change material GSST. (a) Optical micrograph of a thermal probe (NanoFrazor, Swisslitho AG) that offers simultaneous in situ imaging and patterning capabilities. (b) Raman spectra of as-deposited GSST film and the same film in regions patterned with TSPL. (c) Depth map displaying a 100-nm-wide crystalized line resulting from volume contraction upon GSST crystallization. (d) A snapshot cross-sectional temperature profile in a 30-nm-thick GSST film on Si after $300 \mu$ s thermal AFM tip dwell time, simulated using the finiteelement method. This profile corresponds to the maximum crystallization depth for GSST, before going over the melting temperature $(\sim 890 \mathrm{~K})$ on the surface. 
facilitate Joule heating. An alternative AFM-related technique, TSPL, has recently demonstrated its promise for high-resolution lithography processes in nanoelectronics ${ }^{46-49}$ thermochemistry ${ }^{50,51}$ and biomolecular nanodevices. ${ }^{52}$ The approach enables simultaneous in situ patterning and AFM imaging [Fig. 4(a)], and unlike c-AFM, it does not require a closed circuit, making it compatible with a diverse range of substrates. Using this technique, we demonstrated nanoscale patterning of an amorphous GSST film with 30-nm thickness. Micro-Raman measurements confirmed that the thermal AFM writing process leads to local crystallization of GSST, evidenced by a shift in the Raman signal peak from near 160 to $120 \mathrm{~cm}^{-1}$, associated with the crystalline phase of GSST [Fig. 4(b)]. In Fig. 4(c), a TSPL-written 100-nm-wide serpentine line pattern is imaged by tracing the changes of GSST volume upon switching. ${ }^{53}$ The result indicates the potential of the thermal AFM technique for nanoscale patterning of PCMs.

To further elucidate the PCM patterning mechanism, we performed finite-element simulation of the transient temperature profile in GSST during thermal nanopatterning. The model was implemented in the commercial COMSOL heat module. The thermal probe is approximated as a point heat source with $4 \times 10^{-6} \mathrm{~W}$ power following our experimental parameters. Figure 4(d) shows the temperature distribution inside a 30-nm-thick GSST film after a tip-dwelling time of $300 \mu \mathrm{s}$. The highly localized temperature profile suggests that nanoscale patterning with resolution better than $20 \mathrm{~nm}$ is viable with our TSLP technique. The simulation also indicates that only the top $15 \mathrm{~nm}$ of the film is crystallized, largely because the thermally conductive Si substrate serves as an efficient heat sink. By modulating the power on the tip, we envision a modulation on the crystallization depth by reaching different amorphous/crystalline areal distributions, which enables applications such as color depth modulation for grayscale patterning ${ }^{45}$ and continuously phase tuning for holographic ${ }^{54}$ and metasurface applications. ${ }^{42}$ The result validates the potential of TSPL for nanopatterning of PCM ultrathin films, providing a pathway toward realizing topologically optimized, reconfigurable photonic devices.

\subsection{Solution-Deposited Chalcogenide Ge-Sb-Se-Te Films as a Route Toward Low-Loss Phase-Change Optical Materials on Large-Area Nonplanar Platforms}

Although GSST PCMs have been fabricated using either a standard melt-quench (bulk) or thermal evaporation (film/waveguides), an ability to conformally deposit PCM layers on large-area nonplanar platforms such as lenses or substrates with complex surface topography has been increasingly demanded. Employing previously demonstrated methods of dispersing chalcogen-containing alloys into solution, one can form films of these alloys following composition-specific protocols. Solution deposition $(\mathrm{SD})^{55}$ is a cost-effective and scalable method where substrates with virtually any shape and size are amenable to forming conformally coated layers, as illustrated in Fig. 5(a). Here, we discuss the ongoing progress made on processing high-performance $\mathrm{Ge}_{2} \mathrm{Sb}_{2} \mathrm{Se}_{5-x} \mathrm{Te}_{x} \mathrm{PCM}$ alloys, using SD.

Figure 5(b) illustrates the step-wise SD process flow. The process begins with the preparation of bulk $\mathrm{Ge}_{2} \mathrm{Sb}_{2} \mathrm{Se}_{5-x} \mathrm{Te}_{x}$ ( $x=0.5$ to 1.0) formed using a standard melt-quench method. The bulk alloys are ground and filtered through a $120-\mu \mathrm{m}$ sieve to realize fine powder and then dissolved into a homogeneous solvent mixture of ethanethiol (thiol) and ethylenediamine (EDA) ${ }^{56}$ Polished and plasma cleaned $\mathrm{ZnSe}$ substrates are dipped into the solution using a speedcontrolled robotic arm to create films of uniform thickness and surface quality. Finally, the as-coated films undergo soft- and hard-heat treatment to remove residual solvents. To realize optimal performance, SD films must maintain parent alloy stoichiometry, exhibit spatially uniform surface quality, be optically thick $(t \geq 1 \mu \mathrm{m})$, be chemically compatible with the underlying IR transparent substrates, exhibit low optical loss in the mid-wave IR, and convert to desired crystalline phases upon thermal or laser treatment. These key attributes assure minimum degradation to the film's phase change response.

Here, we discuss key process conditions that have deterministic impacts on the resulting quality of coated films. Specifically, the atomic percentages of constituent elements in resulting films are significantly associated with the thiol-EDA mixing ratio. Although $\mathrm{Ge}$ and $\mathrm{Sb}$ elements have been shown to be easily soluble using amine solvents such as propylamine and EDA, ${ }^{57,58} \mathrm{Se}$ and $\mathrm{Te}$ are inherently more challenging to dissolve due to their preferential dissolution kinetics, 
(a)

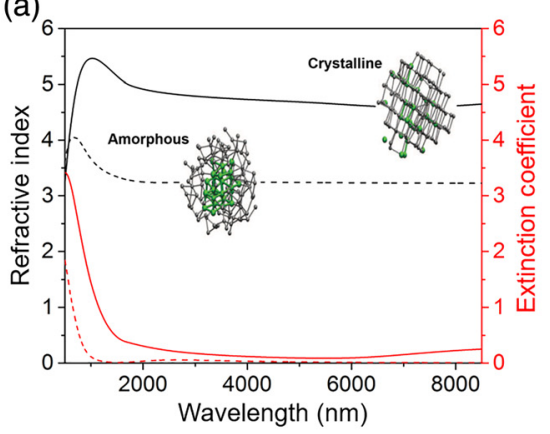

(b)

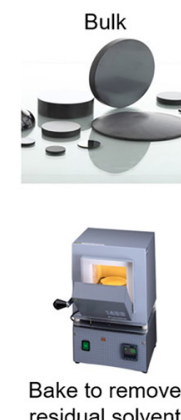

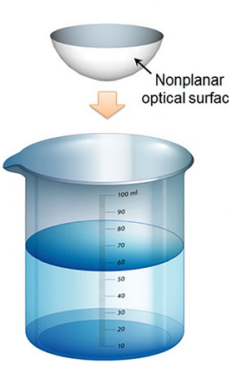

(c)

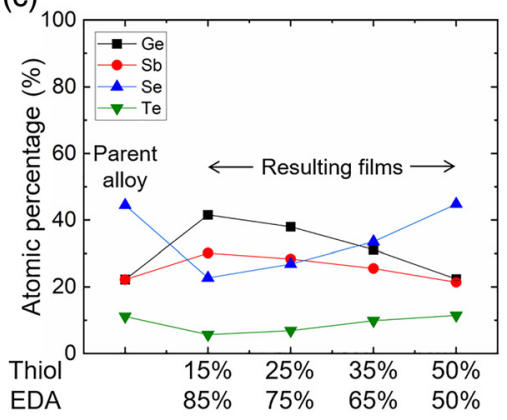

(d) Solvent-only solubility

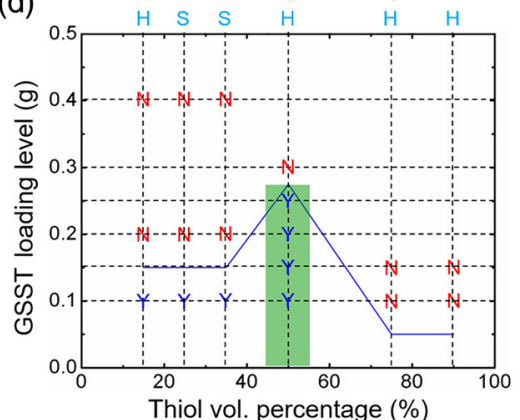

Fig. 5 (a) Contrasts in refractive index and optical loss upon $\mathrm{Ge}_{2} \mathrm{Sb}_{2} \mathrm{Se}_{4} \mathrm{Te}_{1}$ switching and an illustration highlighting that SD enables large-area conformal coating; (b) SD process flow; (c) atomic percentage of constituent elements in resulting $\mathrm{SD} \mathrm{Ge}_{2} \mathrm{Sb}_{2} \mathrm{Se}_{4} \mathrm{Te}_{1}$ films as a function of thiol-EDA mixing ratio and their comparison with a starting parent alloy composition; and (d) optimum chemical process window dictated by intersolvent solubility and solute dissolution.

necessitating the introduction of an additional solvent such as thiol, as shown in prior efforts. ${ }^{56,59}$ Specifically, Te has very low solubility in amine solvents, and thus is partially transferred to a substrate from the solution, disrupting the stoichiometry of resulting films from that of the target parent alloy. This variation, as a function of solvent chemistry, is illustrated in Fig. 5(c). Meanwhile, the composition data extracted using energy dispersive spectroscopy (EDS) on resulting films in Fig. 1(c) indicate that an increase (decrease) in the volume percentage of thiol (EDA) leads to an increase (decrease) in the atomic percentages of $\mathrm{Se}$ and $\mathrm{Te}$ (Ge and $\mathrm{Sb}$ ) in a resulting film. Their atomic percentages match those of a parent alloy when the thiol-EDA mixing ratio becomes 50\% to 50\%. This indicates that the dissolutions of Se/Te and $\mathrm{Ge} / \mathrm{Sb}$ are likely facilitated using thiol and EDA, respectively. To ensure that resulting films maintain the parent alloy stoichiometry in a spatially uniform manner, thiol and EDA are required to be homogeneously mixed. The top $x$ axis of Fig. 5(d) shows intersolvent solubility as a function of thiol-EDA mixing ratio where $\mathrm{H}$ and $\mathrm{S}$ correspond to homogeneous and separated, respectively, indicating the presence of an immiscibility zone across $20 \%$ to $40 \%$ volume percentages of thiol to avoid. The $y$ axis of Fig. 5(d) shows the maximum solute loading level within solutions with varying thiol-EDA mixing ratios where $Y$ and $N$ correspond to yes (complete dissolution) and no (partial dissolution), indicating the maximum loading level achieved at a thiol-EDA mixing ratio of $50 \%$ to $50 \%$, which facilitates material transfer throughput from a parent alloy to a coated film. Therefore, the 50\% to 50\% ratio, represented by the green window in Fig. 5(d), is an optimum condition, which would induce the stoichiometry of resulting films to be spatially homogeneous and consistent with that of a parent alloy while maximizing a film deposition rate.

To assess the phase change behavior of SD films, $\mathrm{Ge}_{2} \mathrm{Sb}_{2} \mathrm{Se}_{4} \mathrm{Te}_{1}$ films underwent in situ grazing-incidence $x$-ray diffraction (GIXRD) at elevated temperatures ranging from room temperature to $325^{\circ} \mathrm{C}$ in an Ar environment, as shown in Fig. 6(a). The GIXRD data collected at room temperature show weak yet noticeable peaks corresponding to Te crystals. Those collected at elevated temperatures up to $325^{\circ} \mathrm{C}$ show the evolution of peaks corresponding to hexagonal $\mathrm{Sb}_{2} \mathrm{Te}_{3}$ crystals, which give rise to an increase in refractive index of the film, ${ }^{19}$ whereas the $\mathrm{Te}$ peaks are carried over to high-temperature data. Given the fact that in situ GIXRD data collected 


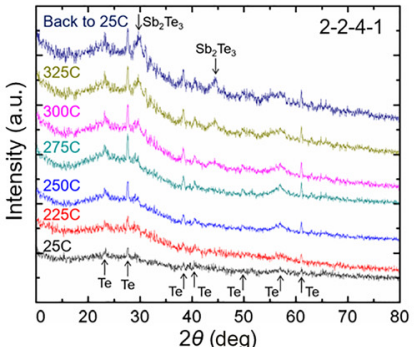

(a)

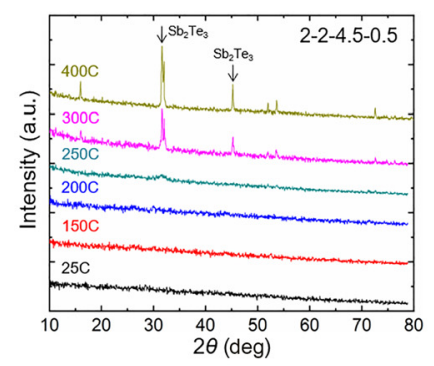

(d)

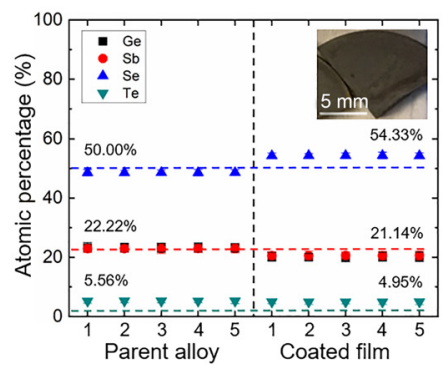

(b)

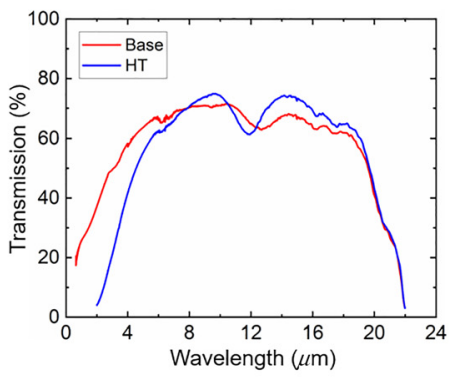

(e)

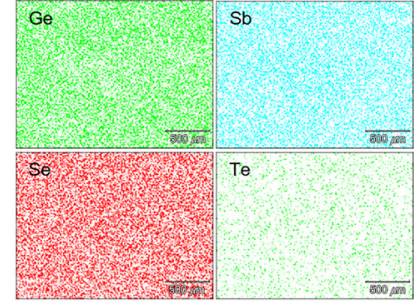

(c)

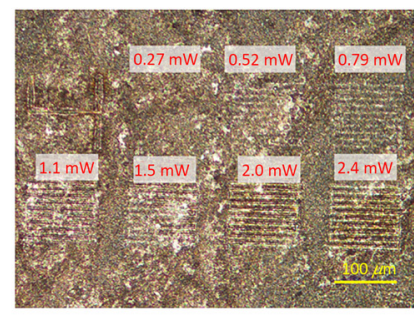

(f)

Fig. 6 (a) In situ GIXRD data of $\mathrm{SD} \mathrm{Ge}_{2} \mathrm{Sb}_{2} \mathrm{Se}_{4} \mathrm{Te}_{1}$ films; (b) EDS-extracted composition of $\mathrm{Ge}_{2} \mathrm{Sb}_{2} \mathrm{Se}_{4.5} \mathrm{Te}_{0.5}$ parent alloys and SD films; (c) EDS-mapped spatial distributions of constituent elements in SD $\mathrm{Ge}_{2} \mathrm{Sb}_{2} \mathrm{Se}_{4.5} \mathrm{Te}_{0.5}$ films; (d) in situ GIXRD data of SD $\mathrm{Ge}_{2} \mathrm{Sb}_{2} \mathrm{Se}_{4.5} \mathrm{Te}_{0.5}$ films; (e) transmission spectra of $\mathrm{SD} \mathrm{Ge}_{2} \mathrm{Sb}_{2} \mathrm{Se}_{4.5} \mathrm{Te}_{0.5}$ films pre- and postheat treatment; and (f) MPL induced modifications on $\mathrm{SD} \mathrm{Ge} \mathrm{Sb}_{2} \mathrm{Se}_{4.5} \mathrm{Te}_{0.5}$ films.

from thermally deposited $\mathrm{Ge}_{2} \mathrm{Sb}_{2} \mathrm{Se}_{4} \mathrm{Te}_{1}$ films show $\mathrm{Sb}_{2} \mathrm{Te}_{3}$ peaks with a relatively higher intensity ${ }^{19} \mathrm{Te}$ crystals existing in SD films are estimated to partially suppress the formation of the desirable $\mathrm{Sb}_{2} \mathrm{Te}_{3}$ crystals upon heat treatment. This necessitates a method to reduce inherent $\mathrm{Te}$ crystals in starting films, thereby promoting the formation of desirable $\mathrm{Sb}_{2} \mathrm{Te}_{3}$ crystals. To achieve this, Te content was reduced from $\mathrm{Ge}_{2} \mathrm{Sb}_{2} \mathrm{Se}_{4} \mathrm{Te}_{1}$ to $\mathrm{Ge}_{2} \mathrm{Sb}_{2} \mathrm{Se}_{4.5} \mathrm{Te}_{0.5}$ in the parent alloys. Figure 6(b) shows the EDS-extracted atomic percentages of constituent elements on various locations of parent alloy, a coated film with the adjusted composition of $\mathrm{Ge}_{2} \mathrm{Sb}_{2} \mathrm{Se}_{4.5} \mathrm{Te}_{0.5}$, and a picture of the sample in the inset. Here, the uniform film coverage, spatial composition homogeneity, and compositional match from a parent alloy to a coated film are apparent, which are further cross confirmed by homogeneous elemental maps at a micron scale, as shown in Fig. 6(c). Figure 6(d) shows that the GIXRD data collected from the $\mathrm{SD} \mathrm{Ge} \mathrm{Sb}_{2} \mathrm{Se}_{4.5} \mathrm{Te}_{0.5}$ films clearly indicate no presence of Te crystals in their as-coated form, while the intensity of $\mathrm{Sb}_{2} \mathrm{Te}_{3}$ peaks is observed to be stronger and sharper, indicating a higher volume fraction and uniform size distribution of the crystals as compared to those from $\mathrm{Ge}_{2} \mathrm{Sb}_{2} \mathrm{Se}_{4} \mathrm{Te}_{1}$ films. The IR transmission spectra (not corrected for Fresnel loss) of the SD films are shown in Fig. 6(e) where both as-coated and heat-treated films exhibit broadband transparency from mid-wave to long-wave IR. However, the short-wave IR transparency noticeably decreases upon heat treatment likely due to $\mathrm{Sb}_{2} \mathrm{Te}_{3}$ crystals-induced optical scattering. Preliminary laser writing to access a tendency toward laser-induced crystallization was carried out on the as-coated film, and visible arrays of patterns on film surfaces illustrate the films' photo sensitivity and phase change, as shown in Fig. 6(f).

In summary, the approach which demonstrates $\mathrm{SD} \mathrm{Ge}_{2} \mathrm{Sb}_{2} \mathrm{Se}_{4.5} \mathrm{Te}_{0.5}$ films as a candidate PCM is presented as a route toward conformal PCM media. Such strategies are expected to greatly increase design flexibility providing new opportunities for optical applications such as reconfigurable metalenses and other advanced devices. As shown, the process' scalability and applicability to diverse composition and substrate material systems is also advantageous. Finally, further insight into the switching behavior of the SD films to understand the limitations in refractive index and electrical conductivity modifications upon either heat treatment or laser exposure will extend integration potential in advanced devices. 


\section{Conclusions}

In this paper, recent advances in material response, methods, and design strategies aimed at improving nanoscale modifications and writing in ChG IR-transparent materials are presented. Multiphoton lithography and etch processing conditions were optimized for creating microstructures in $\mathrm{ChG} \mathrm{As}_{2} \mathrm{~S}_{3}$ films. By incorporating an $\mathrm{AR}$ layer between the $\mathrm{ChG} \mathrm{As}_{2} \mathrm{~S}_{3}$ fabrication layer and the $\mathrm{Si}$ substrate, the standing wave interference was significantly reduced, which resulted in high-quality pillar structures with more controllable dimensions. Gradient effective index nanostructured lenses with diameters of $100 \mu \mathrm{m}$ were fabricated and found to be optically functional. Processing and characterization of high-performance Ge-Sb-SeTe (GSST) PCM alloys is also demonstrated. TSPL was shown to be a good alternative to standard AFM techniques as well as a route to the creation of high-resolution nanostructures by overcoming the optical diffraction limit associated with laser writing in GSST materials. This technique enabled simultaneous in situ patterning and AFM imaging and does not require a closed circuit, making it compatible with a diverse range of substrates. Using TSPL, nanoscale patterning of the ChG PCM GSST with features down to $100 \mathrm{~nm}$ was realized. Solutiondeposited ChG GSST films were shown to be a good route toward low-loss phase-change optical materials on large-area nonplanar platforms. Switching performance and prospects of this system as a functional metalens are also explored with laser exposures. These developments are of importance to the IR materials community as a route to next-generation optical and photonic systems.

\section{Acknowledgments}

This work was funded by the Defense Advanced Research Projects Agency Defense Sciences Office (DSO) Program: EXTREME Optics and Imaging (EXTREME) under Agreement No. HR00111720029.

\section{References}

1. J. L. Adam and X. Zhang, Chalcogenide Glasses: Preparation, Properties and Applications, p. 682, Woodhead Publishing Limited, Cambridge (2014).

2. Y. Zha, M. Waldmann, and C. B. Arnold, "A review on solution processing of chalcogenide glasses for optical components," Opt. Mater. Express 3, 1259-1272 (2013).

3. P. Sharma et al., "Recent developments on the optical properties of thin films of chalcogenide glasses," Prog. Solid State Ch. 44, 131-141 (2016).

4. A. Zakery and S. R. Elliot, Optical Nonlinearities in Chalcogenide Glasses and Their Applications, Springer Berlin, Heidelberg (2007).

5. C. Goncalves et al., "New candidate multicomponent chalcogenide glasses for supercontinuum generation," Appl. Sci. 8, 2082 (2018).

6. Q. Wang et al., "Optically reconfigurable metasurfaces and photonic devices based on phase change materials," Nat. Photonics 10, 60-65 (2016).

7. M. Drigger and C. Rivero-Baleine, "Investigation of the crystallization behavior of laserirradiated EXTREME pattern by Raman spectroscopy," Int. J. Appl. Glas. Sci. 11, 415-420 (2020).

8. C. M. Schwarz et al., "Fabrication and characterization of microstructures created in thermally deposited arsenic trisulfide by multiphoton lithography," J. Micro/Nanolithogr. MEMS MOEMS 16, 023508 (2017).

9. C. M. Schwarz et al., "Multi-photon lithography of $3 \mathrm{D}$ micro-structures in $\mathrm{As}_{2} \mathrm{~S}_{3}$ and $\mathrm{Ge}_{5}\left(\mathrm{As}_{2} \mathrm{Se}_{3}\right) 95$ chalcogenide glasses," Proc. SPIE 9759, 975916 (2016).

10. S. M. Kuebler et al., Frontiers in Optics, San Jose, California (2015).

11. S. Wong et al., "Direct laser writing of three-dimensional photonic crystals with a complete photonic bandgap in chalcogenide glasses," Adv. Mater. 18, 265-269 (2006).

12. J. M. P. Almeida et al., "Sub-wavelength self-organization of chalcogenide glass by direct laser writing," Opt. Mater. 84, 259-262 (2018). 
13. A. Zoubir et al., "Direct femtosecond laser writing of waveguides in $\mathrm{As}_{2} \mathrm{~S}_{3}$ thin films," Opt. Lett. 29, 748-750 (2004).

14. E. Nicoletti et al., "Generation of $\lambda / 12$ nanowires in chalcogenide glasses," Nano. Lett. 11, 4218-4221 (2011).

15. C. M. Schwarz et al., "Fabrication and characterization of micro-structures created by direct laser writing in multi-layered chalcogenide glass," Proc. SPIE 9374, 937403 (2015).

16. C. M. Schwarz et al., "Processing and properties of arsenic trisulfide chalcogenide glasses for direct laser writing of 3D micro structures," Proc. SPIE 8974, 89740P (2014).

17. H. E. Williams, Z. Luo, and S. M. Kuebler, "Effect of refractive index mismatch on multiphoton direct laser writing," Opt. Express 20, 25030-25040 (2012).

18. S. F. Hasan and S. N. Turki, "Design of an antireflection coating for mid-wave infrared regions in the range (3000-5000) nm," Int. J. Innov. Eng. Res. Manag. 2, 96-100 (2013).

19. Y. Zhang et al., "Broadband transparent optical phase change materials for high-performance nonvolatile photonics," Nat. Commun. 10, 4279 (2019).

20. F. De Leonardis et al., "Broadband electro-optical crossbar switches using low-loss $\mathrm{Ge}_{2} \mathrm{Sb}_{2} \mathrm{Se}_{4} \mathrm{Te}_{1}$ phase change material," J. Lightwave Technol. 37, 3183 (2019).

21. Q. Zhang et al., "Broadband nonvolatile photonic switching based on optical phase change materials: beyond the classical figure-of-merit," Opt. Lett. 43, 94-97 (2018).

22. Y. Zhang and J. Hu, "Reconfigurable optics-a phase change for the better," Am. Ceram. Soc. Bull. 99, 36-37 (2020).

23. B. Gholipour et al., "An all-optical, non-volatile, bidirectional, phase-change meta-switch," Adv. Mater. 25, 3050-3054 (2013).

24. J. Rensberg et al., "Active optical metasurfaces based on defect-engineered phase-transition materials," Nano Lett. 16, 1050-1055 (2016).

25. M. J. Dicken et al., "Frequency tunable near-infrared metamaterials based on $\mathrm{VO}_{2}$ phase transition," Opt. Express 17, 18330-18339 (2009).

26. M. Kang et al., "Refractive index patterning of infrared glass ceramics through laser-induced vitrification," Opt. Mater. Express 8, 2722-2733 (2018).

27. M. Kang et al., "Monolithic chalcogenide optical nanocomposites enable infrared system innovation: gradient refractive index (GRIN) optics," Adv. Opt. Mater. 8, 2000150 (2020).

28. A. V. Pogrebnyakov et al., "Reconfigurable near-IR metasurface based on $\mathrm{Ge}_{2} \mathrm{Sb}_{2} \mathrm{Te}_{5}$ phase-change material," Opt. Mater. Express 8, 2264 (2018).

29. C. Koch et al., "Enhanced temperature stability and exceptionally high electrical contrast of selenium substituted $\mathrm{Ge}_{2} \mathrm{Sb}_{2} \mathrm{Te}_{5}$ phase change materials," RSC Adv. 7, 17164 (2017).

30. E. M. Vinod, A. K. Ramesh, and K. S. Sangunni, "Structural transition and enhanced phase transition properties of Se doped $\mathrm{Ge}_{2} \mathrm{Sb}_{2} \mathrm{Te}_{5}$ alloys," Sci. Rep. 5, 8050 (2015).

31. M. Kang et al., "Ultra-low dispersion multicomponent thin film chalcogenide glass for broadband gradient index optics," Adv. Mater. 30, 1803628 (2018).

32. K. Richardson et al., "Advances in infrared GRIN materials: a review," Opt. Eng. 59, 112602 (2020).

33. M. Wuttig, H. Bhaskaran, and T. Taubner, "Phase-change materials for non-volatile photonic applications," Nat. Photonics 11, 465-476 (2017).

34. C. Ríos et al., "Integrated all-photonic non-volatile multi-level memory," Nat. Photonics 9 , 725-732 (2015).

35. C. Rios et al., "Controlled switching of phase-change materials by evanescent-field coupling in integrated photonics," Opt. Mater. Express 8, 2455 (2018).

36. W. Dong et al., "Tunable mid-infrared phase-change metasurface," Opt. Mater. 6, 1701346 (2018).

37. K.-K. Du et al., "Control over emissivity of zero-static-power thermal emitters based on phase-changing material GST," Light Sci. Appl. 6, e16194 (2017).

38. C. Williams et al., "Tunable mid-wave infrared Fabry-Perot bandpass filters using phasechange GeSbTe," Opt. Express 28, 10583 (2020).

39. J. Zheng et al., "GST-on-silicon hybrid nanophotonic integrated circuits: a non-volatile quasi-continuously reprogrammable platform,” Opt. Mater. Express 8, 1551-1561 (2018).

40. C. Wu et al., "Low-loss integrated photonic switch using subwavelength patterned phase change material," ACS Photonics 6, 87-92 (2019). 
41. A.-K. U. Michel et al., "Reversible optical switching of infrared antenna resonances with ultrathin phase-change layers using femtosecond laser pulses," ACS Photonics 1, 833-839 (2014).

42. M. Y. Shalaginov et al., "Reconfigurable all-dielectric metalens with diffraction limited performance," https://arxiv.org/abs/1911.12970 (2019).

43. Y. Zhang et al., "Electrically reconfigurable nonvolatile metasurface using low-loss optical phase change material," https://arxiv.org/abs/2008.06659 (2020).

44. P. Hosseini, C. D. Wright, and H. Bhaskaran, "An optoelectronic framework enabled by low-dimensional phase-change films," Nature 511, 206-211 (2014).

45. C. Ríos et al., "Color depth modulation and resolution in phase-change material nanodisplays," Adv. Mater. 28, 4720-4726 (2016).

46. A. Podpirka et al., "Nanopatterning of GeTe phase change films via heated-probe lithography," Nanoscale 9, 8815-8824 (2017).

47. C. Rawlings et al., "Fast turnaround fabrication of silicon point-contact quantum-dot transistors using combined thermal scanning probe lithography and laser writing," Nanotechnology 29, 505302 (2018).

48. Y. Lisunova et al., "High-Aspect ratio nanopatterning via combined thermal scanning probe lithography and dry etching," Microelectron. Eng. 180, 20-24 (2017).

49. X. Zheng et al., "Patterning metal contacts on monolayer MoS 2 with vanishing Schottky barriers using thermal nanolithography," Nat. Electron. 2, 17-25 (2019).

50. O. Fenwick et al., "Thermochemical nanopatterning of organic semiconductors," Nat. Nanotechnol. 4, 664-668 (2009).

51. D. Pires et al., "Nanoscale three-dimensional patterning of molecular resists by scanning probes," Science 328, 732-735 (2010).

52. X. Liu et al., "High-throughput protein nanopatterning," Faraday Discuss. 219, 33-43 (2019).

53. V. Weidenhof et al., "Atomic force microscopy study of laser induced phase transitions in $\mathrm{Ge}_{2} \mathrm{Sb}_{2} \mathrm{Te}_{5}$," J. Appl. Phys. 86, 5879-5887 (1999).

54. S.-Y. Lee et al., "Holographic image generation with a thin-film resonance caused by chalcogenide phase-change material," Sci. Rep. 7, 1-8 (2017).

55. D. J. Milliron et al., "Solution-phase deposition and nanopatterning of GeSbSe phasechange materials," Nat. Mater. 6, 352-356 (2007).

56. D. H. Webber et al., "Facile dissolution of selenium and tellurium in a thiol-amine solvent mixture under ambient conditions," Chem. Sci., 5, 2498 (2014).

57. M. Waldmann et al., "Structural properties of solution processed $\mathrm{Ge}_{23} \mathrm{Sb}_{7} \mathrm{~S}_{70}$ glass materials," J. Mater. Chem. 22, 17848-17852 (2012).

58. S. Novak et al., "Deposition of $\mathrm{Ge}_{23} \mathrm{Sb}_{7} \mathrm{~S}_{70}$ chalcogenide glass films by electrospray," Thin Solid Films 588, 56-60 (2015).

59. J. Lu et al., "Study of the dissolution behavior of selenium and tellurium in different solvents - a novel route to Se, Te tubular bulk single crystals," J. Mater. Chem. 12, 2755-2761 (2002).

Casey M. Schwarz received her PhD in physics from the University of Central Florida in 2012. She is an assistant professor of physics at Ursinus College. As a postdoctoral researcher, she investigated the processing and properties of innovative materials for future optical device applications. Her research interests now include 3D multi-photon lithography of multicomponent chalcogenide materials, the development and design of optical devices and science, technology, engineering, art, and math outreach.

Stephen M. Kuebler is professor of chemistry and optics at the University of Central Florida (UCF) and founding associate director of the UCF Center for Ethics. He holds joint appointments in the Department of Chemistry and CREOL, The College of Optics and Photonics. His group develops new materials and processes for 3D fabrication by multi-photon lithography and used the technique to fabricate and investigate the novel 3D nanophotonic devices.

Biographies of the other authors are not available. 